\title{
Efeitos do Xilitol associado a produtos fluoretados na desmineralização e remineralização do esmalte dental, uma revisão sistemática
}

\section{Carlos Sampaio de Santana Neto; ${ }^{1}$;na Rita Duarte Guimarães ${ }^{2}$; Silvia Letícia Sena Ferreira $^{3}$ e Hervânia Santana da Costa ${ }^{4}$ \\ 1. Bolsista PIBIC/CNPq, Graduando em Odontologia, Universidade Estadual de Feira de Santana, e-mail: cs.neeto@gmail.com \\ 2. Orientador, Departamento de Saúde, Universidade Estadual de Feira de Santana, e-mail: ardg1999@ gmail.com}

3. Participante do projeto, Departamento de Saúde, Universidade Estadual de Feira de Santana, e-mail: silvialsena@gmail.com

4. Participante do projeto, Departamento de Saúde, Universidade Estadual de Feira de Santana, e-mail: hervacosta@gmail.com

PALAVRAS-CHAVE: Fluoretos; Xilitol; Remineralização Dentária.

\section{INTRODUÇÃO}

As lesões de cárie dentária mostram-se como uma das afecções de maior prevalência na cavidade bucal. A cárie é considerada uma doença complexa, polimicrobiana, uma disbiose (CRUZ et al., 2017). Compostos fluoretados são usados com o objetivo reduzir a progressão da desmineralização dental, em situações de $\mathrm{pH}$ crítico em que há presença de flúor, uma certa parte deste mineral é reposta na apatita formando a fluorapatita (BARATIERI, 2002).

O Xilitol, um adoçante natural não fermentável utilizado em diversos produtos industrializados, na odontologia é utilizado para atuar no lado patológico da cárie através da diminuição da formação do biofilme dental e sua acidogenicidade (STECKSÉN-BLICKS; HOLGERSON; TWETMAN, 2008). Devido a suas características químicas sobre o biofilme dental, esta substância vem sendo pesquisada em associação à produtos fluoretados na tentativa de aumentar a remineralização ou reduzir a desmineralização dental em situações de alto desafio cariogênico, onde o pH do biofilme encontrasse abaixo de 4,5.

Elias, Pinzan e Bastos, (2006) consideram está associação um recurso promissor para a saúde bucal, quando mantidas sobre instrução e motivação em higiene bucal. Este trabalho tem como objetivo identificar através de uma revisão sistemática a literatura presente nas bases de dados PubMed, Lilacs e Cochrane e responder a seguinte pergunta: há evidência científica que suporte o uso clínico da associação de produtos fluoretados ao xilitol?

\section{METODOLOGIA}

Seguindo os princípios da metodologia escolhida, foram selecionados os artigos nas bases de dados PubMed, Lilacs e Cochrane utilizando os seguintes descritores: Fluoride + Xylitol. Os resumos dos estudos foram coletados através do software StArt (State of the Art through Systematic Review) versão 2.3.4.2 e analisados por um pesquisador, o qual aceitava ou recusava um artigo com base na leitura dos resumos e aplicação dos critérios de inclusão e exclusão utilizados no estudo. Os critérios de inclusão foram: artigos na língua inglesa, publicados nos últimos 10 anos, revisões sistemáticas ou ensaios clínicos randomizados, com resumo e texto completo disponíveis na internet. Os 
estudos que não seguiram esses critérios foram excluídos. A construção do artigo seguiu as recomendações PRISMA para revisões sistemáticas.

Os artigos selecionados foram fichados, os ensaios clínicos randomizados foram analisados segundo a lista de informações CONSORT 2010 e a Ferramenta da Colaboração Cochrane para avaliação do risco de viés e as revisões sistemáticas foram analisadas através do checklist PRISMA. As informações encontradas a partir das ferramentas foram utilizadas na tabulação dos dados dos estudos e empregues na construção dos resultados.

\section{RESULTADOS E/OU DISCUSSÃO (ou Análise e discussão dos resultados)}

Foram encontrados 86 estudos durante a pesquisa, destes 77 não se encaixavam aos critérios de inclusão e por isso foram rejeitados, 04 artigos estavam duplicados e 05 foram aceitos. Após a leitura completa e fichamento dos artigos aceitos, foram selecionados 03 estudos que se encaixavam perfeitamente aos critérios de inclusão, destes dois são ensaios clínicos randomizados e um uma revisão sistemática (Imagem 1). Conforme a metodologia, os artigos foram analisados de acordo com a lista de informações CONSORT 2010 e o checklist PRISMA. Foi observado que estes estudos seguem os padrões estabelecidos para a construção de ensaios clínicos randomizados e revisão sistemática.

Não há muitos artigos avaliando a associação de produtos fluoretados ao xilitol e que se encaixem nos critérios metodológicos de uma revisão sistemática. Há que se considerar o alto nível de evidência científica, de acordo com as normas CONSORT e PRISMA e possuem baixo nível de viés.

Estudos clássicos utilizando o xilitol separadamente em gomas de mascar obtiveram resultados positivos na diminuição da quantidade de $S$. mutans (ES) na saliva (ISOKANGAS et al., 1988; KANDELMAN; GAGNON, 1990; SÖDERLING et al., 1989). Gonçalves e colaboradores (2001) e Arunakul e colaboradores (2011) encontraram resultados favoráveis a utilização da associação do xilitol a soluções fluoretadas. Já os resultados de Giertsen, Emberland e Scheie (1999) vão de encontro aos dos artigos citados acima, relatando não ter encontrado diferença significante entre o número de ES na saliva da amostra controle e na saliva da amostra que fez uso de solução fluoretada associada ao xilitol. As informações coletadas por este estudo concordam com o que foi relatado por Giertsen, Emberland e Scheie (1999).

O xilitol pode ser capaz de induzir remineralização em regiões profundas do esmalte, produzidas em zonas de desmineralização com profundidade de 70-80 $\mu$ m equivalente aos estágios iniciais de progressão da cárie, utilizando o efeito do xilitol (MIAKE et al., 2003). Estudos anteriores sugerem que pode haver um efeito sinérgico entre o flúor e o xilitol, podendo estas substâncias ter características aditivas em situações específicas em que as concentrações de xilitol foram de 25 a $35 \%$, sendo que o xilitol pode facilitar a absorção de flúor proveniente de dentifrícios e a absorção de íons cálcio e fosfato da saliva (ARENDS et al., 1990; HICKS; WU; FLAITZ, 2016; SMITS; ARENDS, 1985). Outras pesquisas revelam haver efeitos benéficos na associação de xilitol ao fluoreto de 
sódio mesmo em concentrações baixas de 5 ou $10 \%$ sódio mesmo em concentrações baixas de 5 ou 10\% (SANO et al., 2007; TANGE et al., 2004).
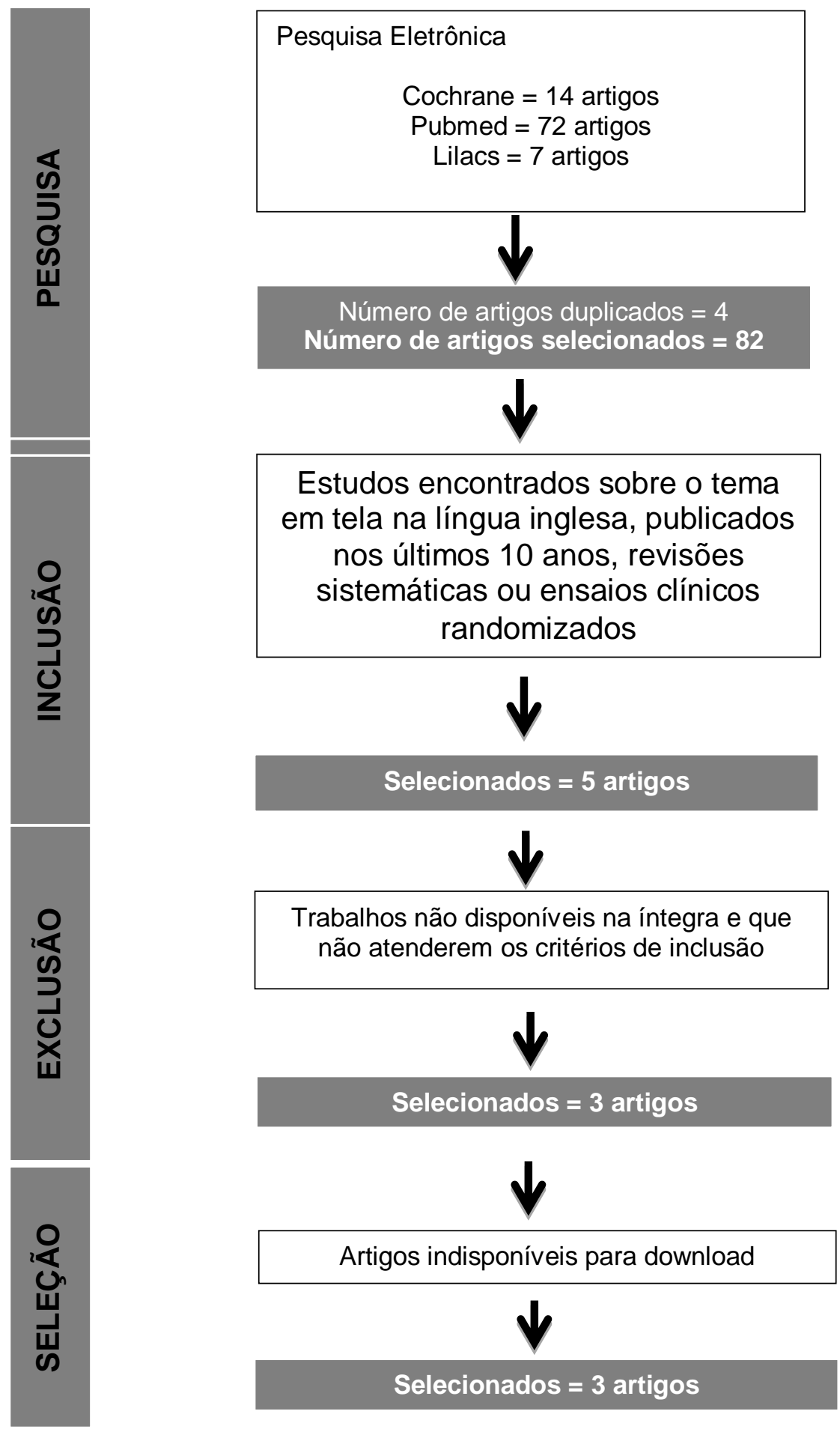

Selecionados $=5$ artigos

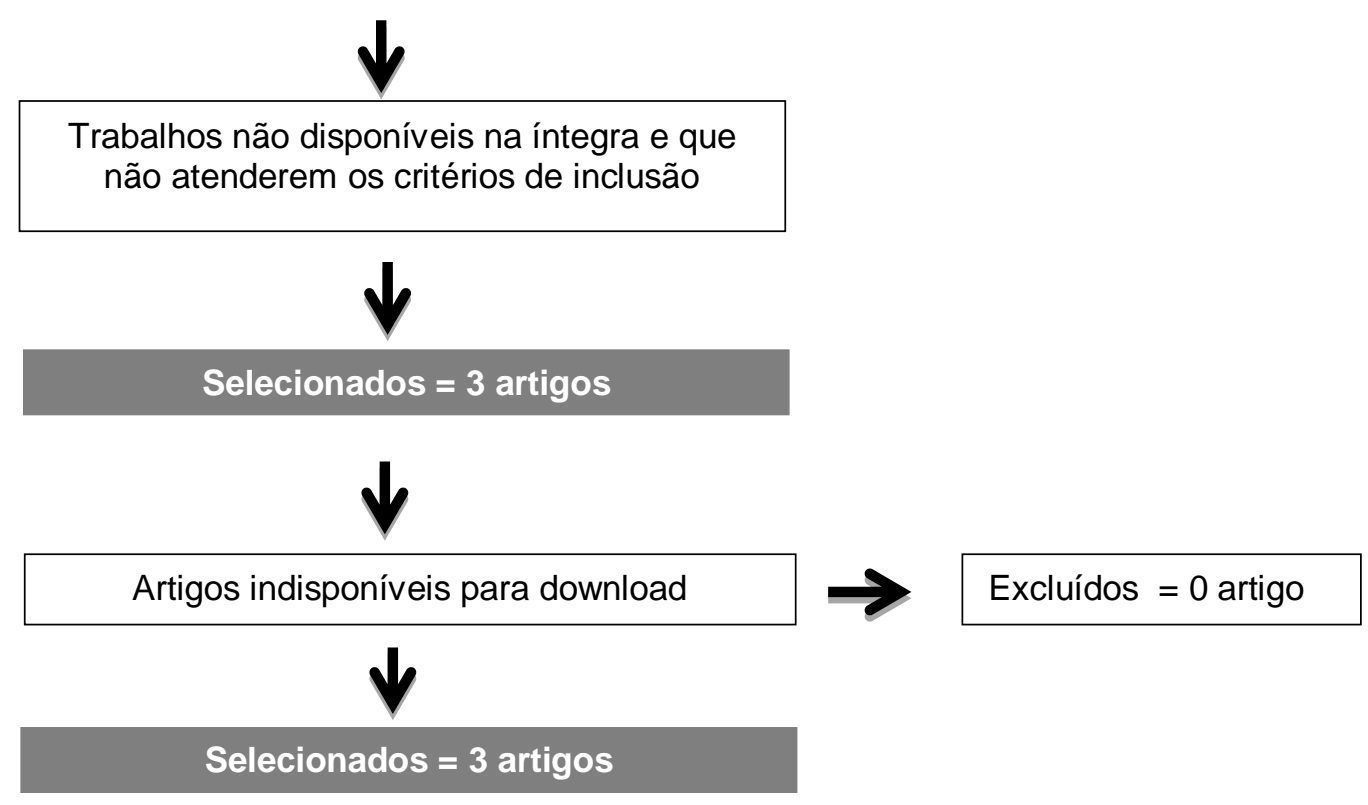

Figura 1 - Fluxograma contendo os métodos de seleção dos artigos.

Apesar dos diversos estudos mostrando que pode haver um efeito sinérgico e aditivo da associação entre os fluoretos e o xilitol, sendo o xilitol um possível potencializador do flúor, os estudos que compõe essa revisão sistemática não concordam com o que foi exposto. Essa divergência de resultados pode ter sido resultado das diferenças metodológicas. Os artigos selecionados foram ensaios clínicos randomizados, enquanto 
que os que demonstraram haver efeito aditivo são in vitro e ex vivo. Além disso, Stecksén-Blicks; Holgerson; Twetman (2008) fizeram uso de comprimidos contendo flúor e xilitol enquanto os estudos comparados utilizam, em sua maioria, dentífricos.

A maior limitação deste estudo foi não ter analisado toda a literatura disponível, sendo que, só foram utilizadas três bases de dados PubMed, Lilacs e Cochrane. Apesar destas bases serem as maiores e mais representativas para pesquisas na área da saúde, especialmente para a construção de revisões sistemáticas, alguns artigos podem não ter sido selecionados por não serem encontrados nas bases de dados utilizadas. Esta situação gera um viés de publicação para o seguinte estudo.

\section{CONSIDERAÇÕES FINAIS}

As bases de dados analisadas apresentam poucos estudos de alta evidência científica sobre o tema deste estudo. De acordo com os artigos analisados, a utilização do xilitol associado a produtos fluoretados não apresentou diferenças significantes em comparação à utilização isolada do fluoreto na incidência de lesões de cárie e nível de $S$. mutans presentes na placa e saliva dos participantes. Recomenda-se que novos estudos como esse sejam reproduzidos englobando mais bases de dados.

\section{REFERÊNCIAS}

ARENDS, J. et al. Combined effect of xylitol and fluoride on enamel demineralization in vitro. Caries Research, v. 24, n. 4, p. 256-257, 1990.

ARUNAKUL, M. et al. Efficacy of xylitol and fluoride mouthrinses on salivary mutans streptococci. Asian Pacific Journal of Tropical Biomedicine, v. 1, n. 6, p. 488-490, 2011.

BARATIERI, L.N. et al. Odontologia restauradora: fundamentos e possibilidades. Säo Paulo, Santos, 2002. p.31-68, ilus, tab. (BR).

CRUZ, L. R. et al. "Cárie é transmissível?" Tipo de informação sobre transmissão da cárie em crianças encontrada através da ferramenta de busca Google®. Revistas, v. 74, n. 1, p. 68, 2017.

ELIAS, F.; PINZAN, A.; BASTOS, J. R. DE M. Influência do complexo flúor-xilitol no controle da placa dentária e do sangramento gengival em pacientes herbiátricos com aparelho ortodôntico fixo. R Dental Press Ortodon Ortop Facial, v. 11, n. 5, p. 42-56, 2006.

GIERTSEN, E.; EMBERLAND, H.; SCHEIE, A. A. Effects of Mouth Rinses with Xylitol and Fluoride on Dental Plaque and Saliva. Caries Research, v. 33, n. 1, p. 23-31, 1999.

GONÇALVES, N. et al. Efeito de soluções fluoretadas contendo xilitol e sorbitol no número de estreptococos do grupo mutans na saliva de seres humanos. Pan Am J Public Health, v. 9, n. 1, p. 30-34, 2001.

HICKS, J.; WU, J.; FLAITZ, C. M. Pediatric Dental Care : Open Access Effect of Xylitol and Fluoride Dentifrices on Human Enamel Surfaces Following Acid-Etching: Scanning Electron Microscopic Study. v. 1, n. 4, p. 1-6, 2016. ISOKANGAS, P. et al. Xylitol chewing gum in caries prevention: a field study in children. Journal of the American Dental Association (1939), v. 117, n. 2, p. 315-320, 1988.

KANDELMAN, D.; GAGNON, G. A 24-month Clinical Study of the Incidence and Progression of Dental Caries in Relation to Consumption of Chewing Gum Containing Xylitol in School Preventive Programs. Journal of Dental Research, v. 69, n. 11, p. 1771-1775, 1990.

MIAKE, Y. et al. Remineralization effects of xylitol on demineralized enamel. Journal of Electron Microscopy, v. 52, n. 5, p. 471-476, 2003.

SANO, H. et al. Effect of Sorbitol, Xylitol, and Xylitol/Sorbitol Chewing Gums on Dental Plaque. Jornal of Oral Science, v. 49, n. 1, p. 67-73, 2007.

SMITS, M. T.; ARENDS, J. Influence of Xylitol- and/or Fluoride-Containing Toothpastes on the Remineralization of Surface Softened Enamel Defects in vivo. Caries Research, v. 19, p. 528-535, 1985.

SÖDERLING, E. et al. Effect of Sorbitol, Xylitol, and Xylitol/Sorbitol Chewing Gums on Dental Plaque. Caries Research, v. 23, p. 378-384, 1989.

STECKSÉN-BLICKS, C.; HOLGERSON, P. L.; TWETMAN, S. Effect of xylitol and xylitol-fluoride lozenges on approximal caries development in high-caries-risk children. International Journal of Paediatric Dentistry, v. 18, n. 3, p. 170-177, 2008.

TANGE, T. et al. The effect of xylitol and fluoride on remineralization for primary tooth enamel caries in vitro.

Pediatric Dental Journal, v. 14, n. 1, p. 55-59, 2004. 